\title{
Relationship Between Structure and Energy of Symmetric Tilt Grain Boundaries in Ag and Ni
}

\author{
A. Ayadi*, H. Laib and O. Khalfallah \\ Laboratory of Microstructure and Defects in Materials (LMDM), \\ Department of Physics, University Constantine 1, Algeria
}

Received: 02.04.2020 \& Accepted: 23.06.2020

Doi: $10.12693 /$ APhysPolA.138.528

*e-mail: aicha.ayadi@umc.edu.dz

\begin{abstract}
The objective of this study is to obtain the correlation between energy and structure of symmetric tilt grain boundaries (STGB) around a [100] axis in Ag and Ni. The atomic simulations use LAMMPS, a parallel molecular dynamics code, with the embedded atom model potential (EAM) to calculate the energies of 200 grain boundaries in FCC bicrystals of Ag and Ni. The results show a similar variation in energy as a function of misorientation for the two metals. This variation has energy minima for $\Sigma 5$ and $\Sigma 13$ boundaries. The grain boundaries structures, described by the structural units model, are of two types. Simple types for the STGB $\Sigma 5$ and $\Sigma 13$ are generally associated with low energies and a complex type for the STGB $\Sigma 65$ is associated with high energies. A pairwise comparison of grain boundary energy ( $\mathrm{Ag}$ vs. $\mathrm{Ni}$ ) shows a correlation between the grain boundary energy and shear modulus which is consistent with the Read and Shockley model
\end{abstract}

topics: symmetric tilt grain boundaries, energy, structure, molecular dynamics (MD)

\section{Introduction}

The grain boundaries affect the mechanical properties of polycrystalline solids. They control the propagation of plastic deformation from one crystal to another, being both an obstacle to the slip of dislocations and a source of dislocations. One of the properties of polycrystalline materials corresponds to the structure of their grain boundarie (GB). This structure is related to the grain boundary geometry and can be described in terms of the structural unit model [1]. Several experimental and atomic simulation studies have been performed to investigate the behavior of grain boundaries energies and their properties [2-6].

In the 1980s, scientists used computational simulation to calculate certain tilt GB energy, with a specific coincidence site lattice being investigated [7]. At present, molecular dynamics (MD) simulation is starting to leave its mark on several researches. In this paper, we use an atomistic simulation method and Large-scale Atomic/Molecular Massively Parallel Simulator (LAMMPS) $[8,9]$ to examine the grain boundary energy and structure of several hundred boundaries in two elemental, facecentered cubic (Fcc) metals: Ag and Ni.

\section{Structure configuration and method}

2.1. Symmetric tilt grain boundary and relaxation

The grain boundaries (GBs) investigated are symmetric tilt grain boundaries (STGBs), with planes $\{0 k l\}$ and misorientation around [100], where grains are rotated by opposite angles $\pm \theta / 2$ around the same [100] axis. We consider more than two hundred STGBs in nickel $(\mathrm{Ni})$ and silver $(\mathrm{Ag})$ and these boundaries are different by their misorientation around the [100] axis. The grain boundary has five degrees of freedom. The conjugate gradient method, as implemented in the MD code LAMMPS [9], generates and relaxes bicrystal structures based on the scheme as in [10-12].

A LAMMPS code is used to perform the simulations and the visualization tool AtomEye has been used for post-processing of the results of the MD simulations.

\subsection{Simulation cell}

The system consists of a simulation box which contains an upper simulation cell, top grain, a lower simulation cell and bottom grain, oriented and attached by the tilt grain boundary. A tilt GB with a specific misorientation or a specific CSL $\Sigma$ value can be built by tilting upper and lower cells [13]. Tilt GBs are obtained by rotating Grain 1 by $+\theta / 2$ and rotating Grain 2 by $-\theta / 2$, along the rotation axis [100]. For example, when $\Sigma 5$ goes with $36.87^{\circ} /$ [100] [031], the orientations of the two grains, top and bottom, along the $X, Y$

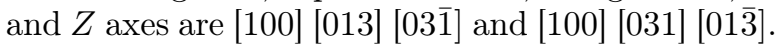
As the boundary conditions are $3 \mathrm{D}$ periodic, this imposes the presence of a half-boundary, so that there are actually two GBs in the simulation box (see Fig. 1). The dimensions of the box are large to avoid interaction between the two grain boundaries. These box sizes are approximately $200 \times 400 \times 10$ 


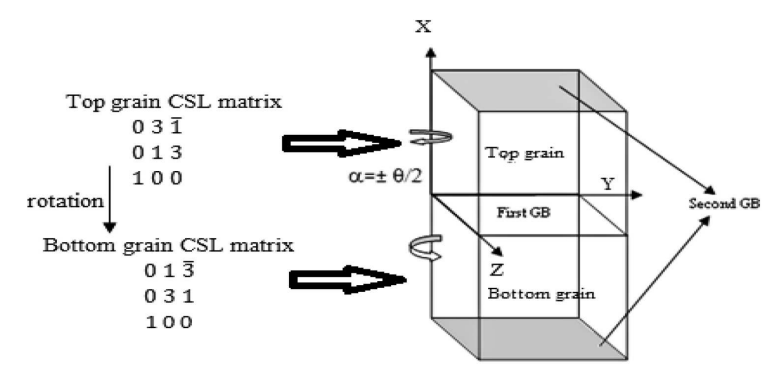

Fig. 1. Tilt grain boundaries model schematic of a $3 \mathrm{D}$ periodic computational cell.

in the $X, Y$ and $Z$ directions, respectively (this size changes slightly depending on the grain orientation) while the minimum distance in the $X$ and $Y$ directions is larger than $8 \mathrm{~nm}\left(a_{\mathrm{Ag}}=0.409 \mathrm{~nm}\right.$ and $a_{\mathrm{Ni}}=0.351 \mathrm{~nm}$ ). The periodic distances along the $X, Y$ and $Z$ axes are related to the rotation axis and misorientation angle (see Fig. 1).

\subsection{Grain boundary energy}

The grain boundary energy is defined as the difference between two energies: the energy of a monocrystalline cell containing $N$ atoms, i.e. $N E_{0}$, and the energy of a bicrystalline cell containing the same number of atoms $E_{1}(\alpha)$. This difference is divided by the area of the grain boundary (Fig. 1). Therefore, the grain energy GE can be calculated in the following way:

$$
E_{\mathrm{JG}}(\alpha)=\frac{E_{1}(\alpha)-N E_{0}}{2 S},
$$

where $2 S$ is the total surface of the two GBs that exist in the cell. It is worth remembering that the use of this approach requires to consider interatomic potentials that are adapted to each metal. Namely, these are the N-body types (EAM) as defined by Foils [14] for Ni and Sheng [15] for Ag.

Table I presents an overview of physical properties of the metals calculated by these potentials.

TABLE I

Some material properties calculated from EAM interatomic potentials for $\mathrm{Ni}[14]$ and $\mathrm{Ag}$ [15].

\begin{tabular}{l|c|c}
\hline \hline & $\mathrm{Ag}$ & $\mathrm{Ni}$ \\
\hline$E_{\text {coh }}[\mathrm{eV}]$ & 2.852 & 4.45 \\
$\gamma_{\mathrm{SF}}\left[\mathrm{mJ} / \mathrm{m}^{2}\right]$ & 22.35 & 128.56 \\
$a[\AA]$ & 4.064 & 3.52 \\
$\mu[\mathrm{GPa}]$ & 16.247 & 39.493 \\
Young's modulus $[\mathrm{GPa}]$ & 109.677 & 180.616 \\
Poisson's ratio $[\mathrm{GPa}]$ & 0.4296 & 0.3980 \\
$C_{11}[\mathrm{GPa}]$ & 131.6395 & 233.2731 \\
$C_{12}[\mathrm{GPa}]$ & 99.1457 & 154.2873 \\
$C_{44}[\mathrm{GPa}]$ & 51.1486 & 127.6365
\end{tabular}

\section{Results and discussion}

\subsection{Energy of symmetric tilt grain boundaries}

Grain boundary energies as a function of the misorientation angle $\theta$ are shown in Figs. 2 and 3, for two metals, Ag and Ni, respectively. Low order coincidence site lattice (CSL) systems are shown as well. The (100) grain boundary energies slightly vary by changing the misorientation angle. The angle $\theta=0^{\circ}$ corresponds to the single crystal and is taken as a reference point for the energy.

In $\mathrm{Ag}$, for low angle STGBs when the misorientation angle increases from 0 to $20^{\circ}$, the energy increases gradually to $800 \mathrm{~mJ} / \mathrm{m}^{2}$. In the range of angles of $20^{\circ}<\theta<70^{\circ}$, the (100) grain boundary energies oscillate between 800 and $1000 \mathrm{~mJ} / \mathrm{m}^{2}$. The curve of Fig. 2a has four minor cusps for $\theta=22.62^{\circ}$, $\theta=36.86^{\circ}, \theta=53.13$ and $\theta=67.38^{\circ}$, which corresponds, respectively, to the (100) STGBs $\Sigma 13$ (015), $\Sigma 5$ (013), $\Sigma 5$ (012) and $\Sigma 13$ (023). These grain boundaries have slightly lower energies than average. These low energy grain boundaries can be qualified as special GBs with respect to their properties. They cannot be considered as representative of all symmetric tilt grain boundaries.
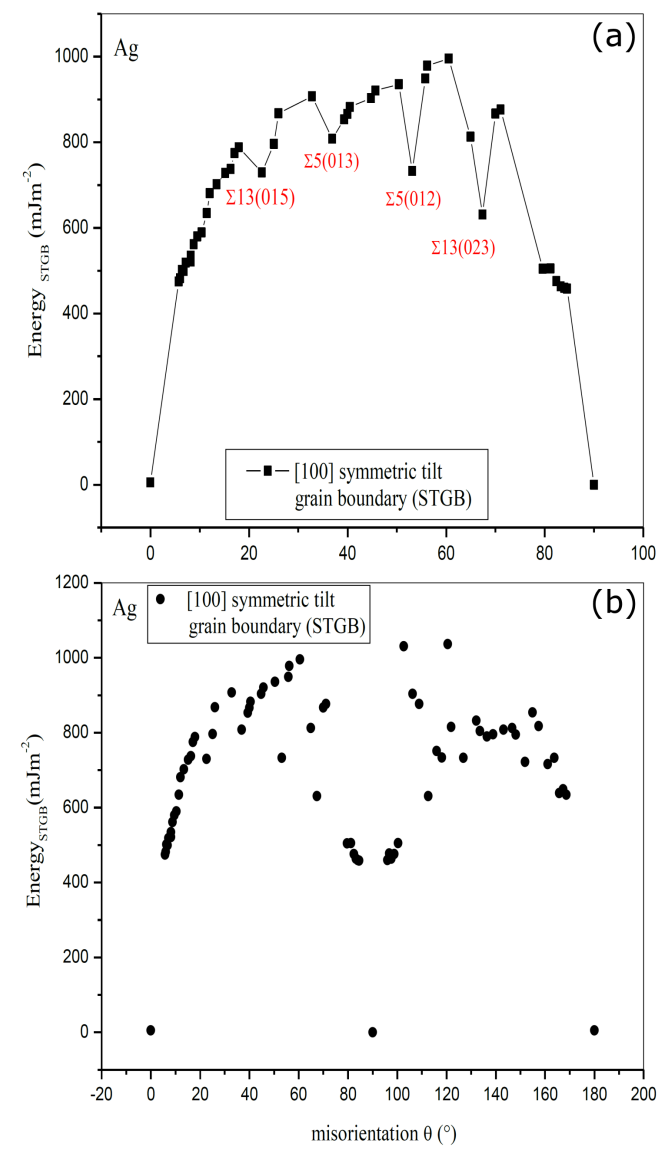

Fig. 2. Symmetric tilt grain boundaries (STGBs) energies as a function of misorientation angle around the (100) axis between two crystals of Ag. (a) $0^{\circ}<\theta<90^{\circ}$; (b) $0^{\circ}<\theta<180^{\circ}$. 

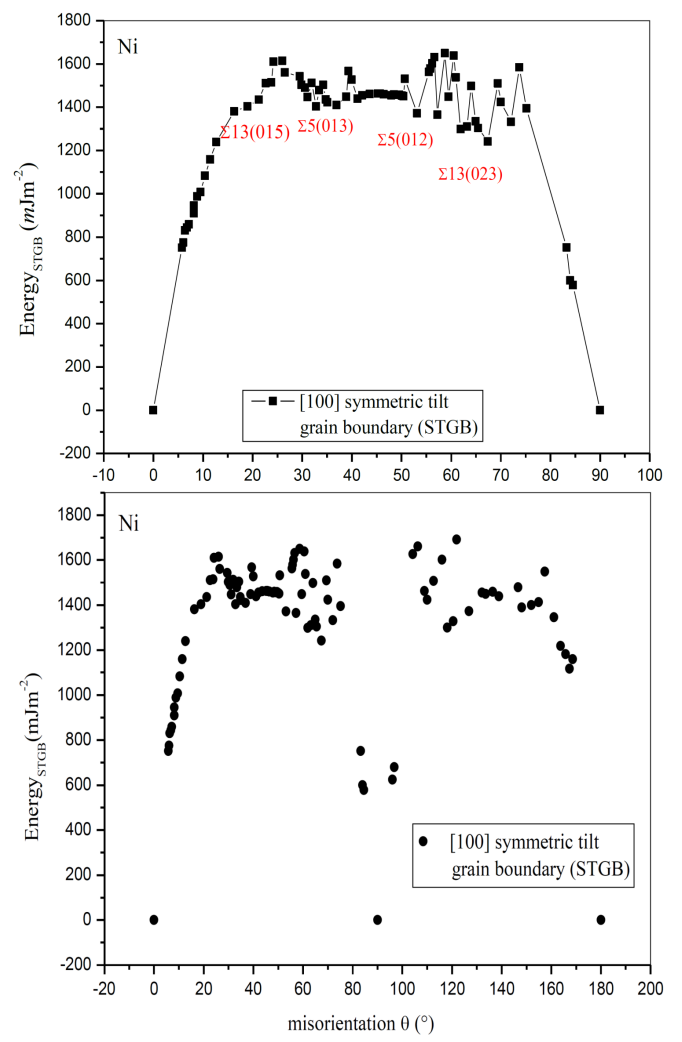

Fig. 3. Symmetric tilt grain boundaries (STGBs) energies as a function of misorientation around the $(100)$ axis between two crystals of Ni. (a) $0^{\circ}<\theta<90^{\circ}$; (b) $0^{\circ}<\theta<180^{\circ}$.

For misorientation angles greater than $70^{\circ}$, the (100) grain boundary energies decrease and vanish for $\theta=90^{\circ}$. Figure $2 \mathrm{~b}$ has a symmetry with respect to $90^{\circ}$ which corresponds to the properties of symmetry of the cubic structures in the rotations around the [100] axis.

Figure 3 shows the same shape as Fig. 2 of the variation of STGBs energies as a function of misorientation in $\mathrm{Ni}$. When the angle increases from $0^{\circ}$ to $20^{\circ}$, the energy increases to reach about $1400 \mathrm{~mJ} / \mathrm{m}^{2}$, at $\theta=20^{\circ}$. In the range $20^{\circ}<\theta<70^{\circ}$, the majority of grain boundaries have energies between 1400 and $1700 \mathrm{~m} / \mathrm{Jm}^{2}$. Energy minima have values slightly lower than the average, which corresponds to the angles $\theta=22.62^{\circ}$, $\theta=36.86^{\circ}, \theta=53.13^{\circ}$ and $\theta=67.38^{\circ}$ which corresponds respectively to the STGBs $\Sigma 13$ (015), $\Sigma 5$ (013), $\Sigma 5$ (012) and $\Sigma 13$ (023).

For angles of disorientation greater than $70^{\circ}$, the energy of the grain boundary decreases to zero at $90^{\circ}$. Symmetry of $90^{\circ}$ describes the symmetrical properties of cubic structures in rotations around the (100) axis, see Fig. 3b. For the two metals, $\mathrm{Ni}$ and $\mathrm{Ag}$, computer modeling of STGBs presents a variation of energy as a function of misorientation, which was already examined by several researchers in metals $\mathrm{Al}, \mathrm{Cu}[7,11,16]$, Mo [17] having cubic structure CC, and in Fe [18] of structure type FCC.

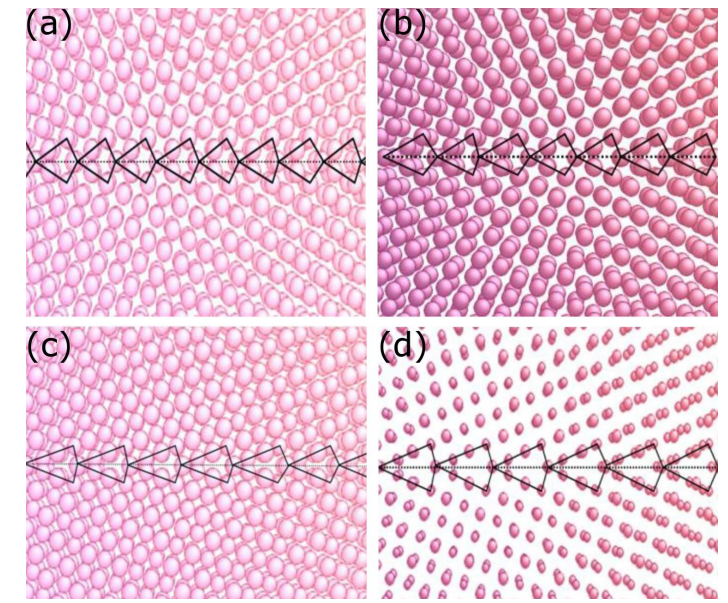

Fig. 4. Atomic structure of STGB $\Sigma 5$ from structural units of AAAAA type. Images viewed by AtomEye. $\Sigma 5$ (012) $\theta=53.13^{\circ}$ for (a) $\mathrm{Ag}$ and (b) Ni. $\Sigma 5$ (013) $\theta=36.86^{\circ}$ for (c) $\mathrm{Ag}$ and (d) Ni.

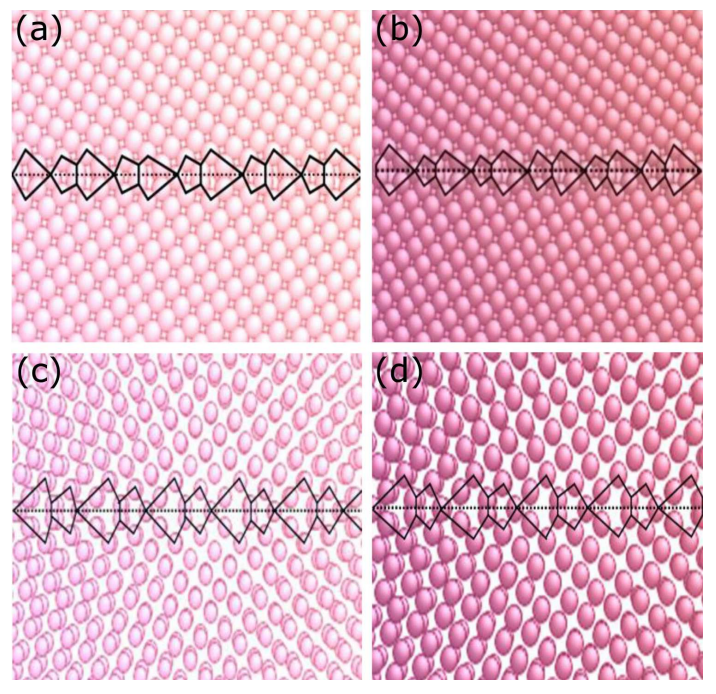

Fig. 5. Atomic structure of STGB $\Sigma 13$ from structural units of $\mathrm{CBCBCB}$ type. Images viewed by AtomEye. $\Sigma 5$ (013) $\theta=22.62^{\circ}$ for (a) $\mathrm{Ag}$ and (b) Ni. $\Sigma 5$ (023) $\theta=67.38^{\circ}$ for (c) $\mathrm{Ag}$ and (d) $\mathrm{Ni}$.

The intergranular energies for nickel are higher than those for silver which, as expected, has given the force of stronger atomic bonds in $\mathrm{Ni}$, and similarly for the stacking fault energies, see Table I.

\subsection{Atomic structure of grain boundaries: model of structural units}

In the model of the structural unit, the grain boundary structure can be described using the polyhedral [1]. The polyhedral unit model is present as the segregation sites for small interstitial solute atoms [19, 20].

The relationship between the GB energies and structures is associated with the coincidence site lattice model as well. The structures visualized, in Figs. 4 and 5, for silver and nickel show that 


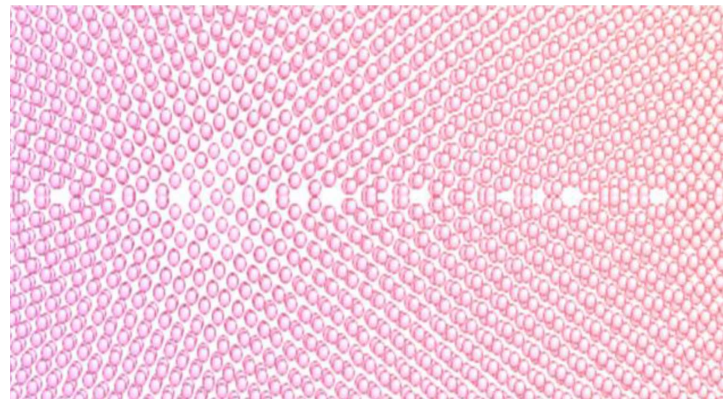

Fig. 6. Complex atomic structure of the symmetric tilt boundary $\Sigma 65$ (047) in silver.

the structure of the symmetric tilt grain boundaries (STGBs) $\Sigma 5(012)$ and $\Sigma 5(013)$ consists of a single simple structural unit of type A. For STGBs $\Sigma 13$ (015) and $\Sigma 13$ (023), the images show two different structural units of type B and C. These grain boundaries correspond to energy minima. The same unit-cell polyhedral units are shown in detail by A.D. Banadaki et al. [21] in FCC metals. The structure of the grain boundaries by the model of the structural units becomes more complex in the case of the symmetrical flexural joint $\Sigma 65$ (047), see Fig. 6.

\subsection{Relationship between grain boundary energy and physical parameters of materials}

Figure 7 shows pairwise comparisons of STGBs energies for $\mathrm{Ag}$ and $\mathrm{Ni}$. Black dots represent the energy of the same (100) STGBs (same macroscopic structure) in Ag and Ni. These points fall on a line that passes through the origin, indicating a strong correlation between the grain boundary energies in each metal.

We have multiplied the parameters listed in Table I by the appropriate power of the lattice parameter so as to obtain the same unit as grain boundary energy $\left(\mathrm{J} / \mathrm{m}^{2}\right)$, in order to have the link between the grain boundary energies of each metal with these properties (stacking fault energy $\gamma_{\mathrm{SF}}$, the shear modulus $\mu$ and the $C_{44}$ elastic constant).

The regression lines resulting from the ratios $\gamma_{\mathrm{SF}}$, $\mathrm{a}_{44}$ and $\mu$ are illustrated in Fig. 7 in blue, green and red colors, respectively. The grain boundary energies are arranged with the ratio of the shear modulus a $\mu$ and the ratio of $\mathrm{aC}_{44}$.

The black dots of the grain boundary energy and the line (green) of the shear modulus ratio present a perfect agreement. The relation between the grain boundary energy and the shear modulus is compatible with the dislocation model of the grain boundaries structure where the elastic energy of a dislocation is proportional to the shear modulus which corresponds to the Read and Shockley model for the energies of low angle misorientation of GB [22] and also observed for the high angle misorientation boundaries [23-24].

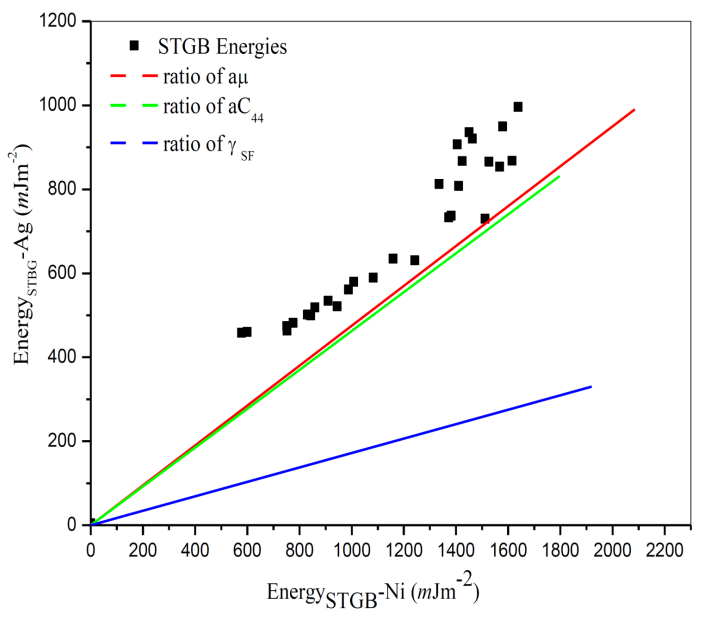

Fig. 7. Comparison of grain boundary energies. Data points represent pairs of boundaries that share the same macroscopic structure. Lines show the scaling predicted by various material parameters $\mu, C_{44}$ and $\gamma_{\mathrm{SF}}(\mathrm{Ag}$ vs $\mathrm{Ni}$ ).

The stacking fault energy does not correlate with the STGBs energies around the [100] axis which has been shown in several studies except for a few special cases [16].

\subsection{Correlation structure - energy}

The structure and energy of grain boundaries have been approached from several perspectives. The symmetric tilt grain boundaries (STGB) which have minimum energies have a periodic structure with a short period and a simple model of the structural units, case of the symmetric tilt boundaries $\Sigma 5$ (012), $\Sigma 5$ (013), $\Sigma 13$ (015) and $\Sigma 13$ (023). Conversely, when the boundaries have strong energies, the structure becomes more complex with a very long periodicity.

In the STGB, the structure is of the general AAABAAAAB type where units B correspond to the cores of the secondary intrinsic dislocations accommodating the gap at the coincidence of the AAAA boundaries. Secondary dislocations have been shown to be linked to structural units with the grain boundary structure [25]. This confirms the relationship between the grain boundary energies and the shear modulus where this energy is identified with the model of the dislocations of the grain boundary structure where the elastic energy of a dislocation is proportional to the shear modulus which corresponds to the Read and Shockley model.

The energy intensity of the grain boundaries is controlled by the stacking fault energy or by the cohesion energy of the considered metal.

\section{Conclusion}

The grain boundary energies are stronger for nickel. This was predictable given the strong cohesive energy and stacking fault energy. 
The variation of symmetric tilt grain boundary energies as a function of misorientation around the (100) axis is similar for the two metals, Ni and Ag. This type of variation has been observed for other bi-crystals with several metals with the CFC structure, such as $\mathrm{Al}$ and $\mathrm{Cu}$.

Boundaries which have energy minima for particular parameter values, $\Sigma, \Sigma 5$ and $\Sigma 13$, can be qualified as "special" boundaries and could have particular properties.

The structure of the STGB is described by the presented model of the structural units:

- the low-energy boundaries have good agreement structure and short period, they are described by a single unit of type A for the $\Sigma 5$ boundaries, and two units, $\mathrm{B}$ and $\mathrm{C}$, for the $\Sigma 13$ boundaries,

- the high energy boundaries have a complex structure of bad arrangement and a very long period in terms of structural units.

The pairwise comparison of the grain boundary energies of silver and nickel shows a strong correlation. The grain boundaries energies are aligned with the ratio of the two shear moduli $\mathrm{a} \mu(\mathrm{Ag}) / \mathrm{a} \mu(\mathrm{Ni})$ and the ratio of the elastic constants $\mathrm{a}_{44}(\mathrm{Ag}) / \mathrm{a} C_{44}(\mathrm{Ni})$. This arrangement shows a relationship between the grain boundary energies and the shear modulus. The dislocation model of the grain boundary structure where the elastic energy of a dislocation is proportional to the shear modulus is consistent with the Read and Shockley model.

\section{References}

[1] M.F. Ashby, F. Spaepen, S. Williams, Acta Metall. 26, 1647 (1978).

[2] P. Mondal, A. Klein, W. Jaegermann, H. Hahn, Solid State Ionics. 118, 331 (1999).

[3] C. Cheung, U. Erb, G. Palumbo, Mater. Sci. Eng. A 185, 39 (1994).

[4] H. Kokawa, J. Mater. Sci. 40, 927 (2005).

[5] S.E. Babcock, J.L. Vargas, Ann. Rev. Mater. Sci. 25, 193 (1995).

[6] M. Frary, C.A. Schuh, Appl. Phys. Lett. 83, 3755 (2003).

[7] D. Wolf, Acta Mater. 37, 1983 (1989).
[8] S. Plimpton, J. Comput. Phys. 117, 1 (1995).

[9] S. Plimpton, P. Crozier, A. Thompson, Large-scale Atomic/Molecular Massively Parallel Simulator, Sandia National Laboratories, 2007.

[10] M.A. Tschopp, S.P. Coleman, D.L. McDowell, Integ. Mater. Manuf. Innov. 4, 176 (2015).

[11] M.A. Tschopp, D.L. McDowell, Philos Mag. 87, 3871 (2007).

[12] M.A. Tschopp, G.J. Tucker, D.L. McDowell, Acta Mater. 55, 3959 (2007).

[13] H. Zheng, X-G. Li, R. Tran, C. Chen, M. Horton, D. Winston, K.A. Persson, S.P. Ong, Acta Mater. 186, 40 (2020).

[14] S.M. Foiles, M.I. Baskes, M.S. Daw, Phys. Rev. B 33, 7983 (1986).

[15] H.W. Sheng, M.J. Kramer, A. Cadien, T. Fujita, M.W. Chen, Phys. Rev. B 83, 134118 (2011).

[16] E.A. Holm, D.L. Olmsted, S.M. Foiles, Scr. Mater. 63, 905 (2010).

[17] S. Ratanaphan, D.L. Olmsted, V.V. Bulatov, E.A. Holm, A.D. Rollett, G.S. Rohrer, Acta Mater. 88, 346(2015).

[18] Y. Shibuta, S. Takamoto, T. Suzuki, ISIJ Int. 48, 1582 (2008).

[19] X. Zhou, D. Marchand, D.L. McDowell et al., Phys. Rev. Lett. 116, 075502 (2016).

[20] C.L. Briant, R.P. Messmer, Acta Metall. 30, 1811 (1982).

[21] A.D. Banadaki, S. Patala, NPJ Comput. Mater. 3, 13 (2017).

[22] W.T. Read, W. Shockley, Phys. Rev. 78, 275 (1950).

[23] A.P. Sutton, R.W. Balluffi, Interfaces in Crystalline Materials, Clarendon Press, Oxford 1995.

[24] K.T. Aust, B. Chalmers, Energies and Structure of Grain Boundaries in Metal Interfaces, ASM, Cleveland (OH) 1952.

[25] O. Hardouin Duprac, S. Poulat, A. Larère, J. Thibault, L. Priester, Phil. Mag. A 80, 853 (2000). 